\title{
Diversity and specificity of microsatellites within Aspergillus section Fumigati
}

Ricardo Araujo ${ }^{1,2^{*}}$, António Amorim ${ }^{1,2}$ and Leonor Gusmão ${ }^{1}$

\begin{abstract}
Background: Microsatellites (or short tandem repeats, STRs) are the genetic markers of choice for studying Aspergillus fumigatus molecular epidemiology due to its reproducibility and high discrimination power. However, the specificity of these markers must be investigated in a group of isolates from closely related species. The aim of this work was to test a microsatellite-based PCR multiplex previously designed for A. fumigatus in a set of species belonging to section Fumigati, namely Aspergillus fumigatiaffinis, Aspergillus lentulus, Aspergillus novofumigatus, Aspergillus unilateralis, Aspergillus viridinutans, Neosartorya fischeri, Neosartorya hiratsukae, Neosartorya pseudofischeri and Neosartorya udagawae.
\end{abstract}

Results: The reference A. fumigatus strain ATCC 46645 was easily genotyped in standard conditions showing a final electrophoretic profile of 8 expected peaks corresponding to each microsatellite locus. Inversely, no peaks were observed for all other species from section Fumigati, with an exception for marker MC6b in A. unilateralis. By screening the genome sequence of Neosartorya fischeri NRRL 181, the results showed that MC3, MC6a and MC7 might be employed for $N$. fischeri genotyping since these markers present several repeats of each motif. The accumulation of insertions and deletions was frequently observed in the genomic regions surrounding the microsatellites, including those where the A. fumigatus primers are located. The amplification of microsatellite markers in less stringent amplification conditions resulted in a distinct electrophoretic profile for species within section Fumigati.

Conclusions: Therefore, the microsatellite-based PCR multiplex allow simple identification of A. fumigatus and, with a slight modification of temperature conditions, it also allows discriminating other pathogenic species within section Fumigati, particularly A. fumigatiaffinis, N. fischeri and N. udagawae.

Keywords: Aspergillus fumigatus, Invasive aspergillosis, Molecular identification, Multiplex PCR, Short tandem repeats, Microsatellites

\section{Background}

Molecular diagnosis of fungal diseases has become increasingly more used in clinical laboratories and new species morphologically similar to Aspergillus fumigatus were surprisingly revealed [1,2]. Section Fumigati includes fungal species closely related to A. fumigatus that can go from the anamorphous Aspergillus species to the teleomorphic species of the genus Neosartorya [3]. Misidentification of fungal species within section

\footnotetext{
* Correspondence: ricjparaujo@yahoo.com

'IPATIMUP, Institute of Molecular Pathology and Immunology, University of Porto, Rua Dr. Roberto Frias, s/n, 4200-465 Porto, Portugal

${ }^{2}$ Faculty of Sciences, University of Porto, Rua do Campo Alegre $s / n$, 4169-007 Porto, Portugal
}

Fumigati was sporadically reported in some laboratories, particularly of fungal isolates afterwards identified as Aspergillus lentulus, Aspergillus viridinutans, Aspergillus fumigatiaffinis, Aspergillus fumisynnematus, Neosartorya pseudofischeri, Neosartorya hiratsukae and Neosartorya udagawae $[1,2,4,5]$. These species present similar microscopical and macroscopical features to A. fumigatus and, therefore, molecular identification is at present recommended for the correct identification of species within section Fumigati. A set of genes, namely actin, calmodulin, internal transcribed spacer (ITS), rodlet A and/or $\beta$ tubulin, has been proposed for a correct identification of A. fumigatus and related species following sequencing analysis [3,6]. Multilocus sequence typing (MLST) [4], random amplified polymorphic DNA [7], restriction

\section{() Biomed Central}

(c) 2012 Araujo et al.; licensee BioMed Central Ltd. This is an Open Access article distributed under the terms of the Creative Commons Attribution License (http://creativecommons.org/licenses/by/2.0), which permits unrestricted use, distribution, and reproduction in any medium, provided the original work is properly cited. 
fragment length polymorphism [8] and microspherebased Luminex assay [9] may allow molecular identification of A. fumigatus. Recently, a practical and cheap electrophoretic strategy was described for molecular identification of $A$. fumigatus and distinction of the species within the section Fumigati [10].

In addition to fungal identification, genotyping methodologies have been improving and gained importance in clinical laboratories. Microsatellite typing methods are very useful for studying $A$. fumigatus molecular epidemiology due to its reproducibility and high discrimination power (around 0.9997). A group of eight microsatellite markers combined in a single PCR multiplex assay with high discrimination power is currently available for $A$. fumigatus genotyping [11]. Such tool may be very useful to investigate outbreaks in clinical units, to evaluate quality control programmes particularly in units admitting critical-care patients, to identify patients with chronic fungal colonization (e.g. some cystic fibrosis patients) and patients with invasive disease caused by multiple fungal strains [11-14]. In addition, genotyping approaches might allow evaluating the response of patients to the antifungal therapies [12].

Few microsatellites (or short tandem repeats - STRs) have been described as species-specific [15-18], while others are transversal to a group of closely related species [19]. Nevertheless, these markers are of extreme utility for population and conservation genetics. The complete genome sequence of Neosartorya fischeri, a sibling species, was recently published and high homology was revealed when comparing to A. fumigatus. Repeat elements density was very similar when comparing these two species and two strains of A. fumigatus [20]. The genomic dynamics for acquisition and removal of microsatellites in closely related species is still unknown, and therefore, it is of scientific relevance to compare and highlight the diversity of some microsatellites in a group of very closely related fungi.

Aspergillus fumigatus is one of the most common agents of systemic mold infections. Genotyping strategies (mostly employing microsatellites) have been described as very useful in labs for detection of outbreaks, identification of patients chronically colonized with A. fumigatus and monitoring of antifungal efficacy in patients $[2,5]$. In addition, sibling species within section Fumigati should also be promptly identified as they present considerable differences in antifungal resistance
[21]. The specificity of microsatellite-based PCR multiplex to A. fumigatus was first confirmed in a group of Aspergillus species [11], but it is also important to assess both the specificity and the diversity of these microsatellites within Aspergillus section Fumigati. Therefore, the two aims of this study were to evaluate the specificity of a set of previously described microsatellite markers to A. fumigatus [11] in a group of closely-related species and the ability of the multiplex to identify $A$. fumigatus and other species belonging to section Fumigati based on the presence/absence of some microsatellite markers.

\section{Results}

Standard microsatellite-based multiplex PCR tested with Aspergillus spp. and Neosartorya spp

A set of eight microsatellites previously described for $A$. fumigatus genotyping strategy was tested with other species belonging to Aspergillus section Fumigati, namely A. fumigatiaffinis, A. lentulus, A. novofumigatus, A. unilateralis, A. viridinutans, $N$. fischeri, $N$. hiratsukae, $N$. pseudofischeri, and N. udagawae, and a reference strain of $A$. fumigatus. The reference A. fumigatus ATCC 46645 was easily genotyped with the standard multiplex conditions and a profile of eight peaks was produced after electrophoretic separation, each one corresponding to a single microsatellite (see Additional file Figure A1). Similar profile was observed for the remaining ten isolates of A. fumigatus, as previously described $[11,12]$. A similar approach was followed for non-fumigatus fungal isolates. No specific PCR amplification products were observed for all tested species from section Fumigati, with the exception of MC6b in A. unilateralis. Sequence analysis of MC6b in A. unilateralis confirmed that this genomic sequence was similar to the sequence of $A$. fumigatus (Figure 1), therefore excluding unspecific amplification of other genomic regions. Nevertheless, the multiplex conditions previously described for $A$. fumigatus genotyping proved to be highly specific, even with the amplification of MC6b in A. unilateralis, as the set of eight microsatellite markers could be uniquely observed in A. fumigatus isolates.

\section{Microsatellites in A. fumigatus AF293 versus N. fischeri NRRL 181}

We screened the complete genome sequence of $N$. fischeri NRRL 181 in order to locate and compare the



Figure 1 Alignment of the marker MC6b sequences in Aspergillus fumigatus and Aspergillus unilateralis. 
microsatellite markers employed for A. fumigatus genotyping. Few microsatellites previously described in A. fumigatus were also found in $N$. fischeri genome, with a single one having more than 30 repetitive motifs (e.g. MC3), while other genomic regions were found more stable without the ability to accumulate repeats. Markers MC3, MC6a and MC7 showed sequences with more than three repeats of the original motif detected in A. fumigatus, representing microsatellites that are potentially polymorphic and might be employed for $N$. fischeri genotyping. Figure 2 shows a set of eight genomic sequences in $N$. fischeri previously described to be unstable in A. fumigatus, representing microsatellites. Curiously, the accumulation of insertions and deletions in these genomic regions was frequently observed, including the regions where the $A$. fumigatus primers were located. Thus, some markers are not expected to be amplified in $N$. fischeri due to extensive modifications of primer regions in the genome of this fungus, as it is the case of MC3, MC1 and $\mathrm{MC} 8$ forward primers and $\mathrm{MC} 2$ reverse primer (Figure 2).

\section{Microsatellite-based PCR multiplex for identification of fungal species}

We have confirmed the specificity of the microsatellite multiplex for A. fumigatus within section Fumigati with a single exception observed in $A$. unilateralis (marker MC6b). However, it could not be discarded the detection of few other markers in species belonging to section Fumigati if less stringent PCR conditions were employed, as some markers were found in the genome of $N$. fischeri NRRL 181. Therefore, we had tested distinct amplification temperatures (from 48 to $60^{\circ} \mathrm{C}$ ) in the group of species belonging to section Fumigati. Few markers could be amplified after decreasing the PCR annealing temperature from $60^{\circ} \mathrm{C}$ to $55^{\circ} \mathrm{C}$ (see Table 1 ). Eight peaks previously observed in A. fumigatus were similarly found when testing less stringent PCR conditions. Sequencing analysis of those amplicons revealed genomic similarities to $A$. fumigatus (see Additional file Table A1; a single exception was MC3 primers that amplified an unspecific region). Remarkably, distinct electrophoretic profiles were obtained for all tested species based on the amplification of the microsatellite

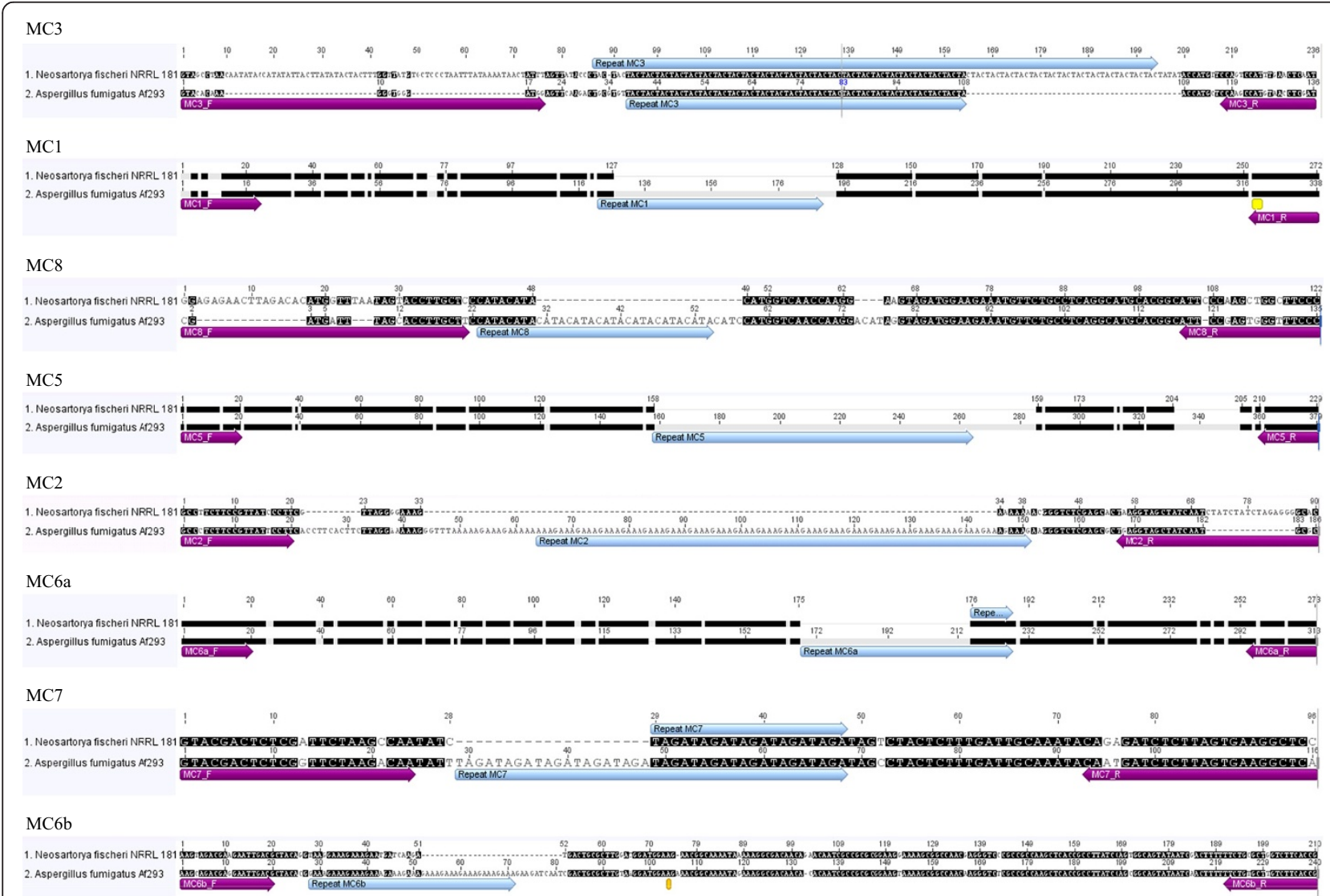

Figure 2 Alignment of eight microsatellites sequences in Neosartorya fischeri NRRL 181 andAspergillus fumigatus AF293 (similar nucleotides in both sequences are marked black while polymorphic sequences are marked white). The primers proposed for microsatellite amplification are marked, as well as the microsatellites within the sequence. 
Table 1 List of markers amplified at $55^{\circ} \mathrm{C}$ annealing temperature in the group of species belonging to section Fumigati

\begin{tabular}{|c|c|c|c|c|c|c|c|c|}
\hline & MC3 & MC1 & MC8 & MC5 & MC2 & MC6a & MC7 & MC6b \\
\hline Aspergillus fumigatus ATCC 46645 & $\sqrt{ }$ & $\sqrt{ }$ & $\sqrt{ }$ & $\sqrt{ }$ & $\sqrt{ }$ & $\sqrt{ }$ & $\sqrt{ }$ & $\sqrt{ }$ \\
\hline Aspergillus fumigatiaffinis CBS 117186 & $\sqrt{ }^{a}$ & & & $\sqrt{ }$ & & & & $\sqrt{ }$ \\
\hline Aspergillus lentulus CBS $116880^{\mathrm{b}}$ & $\sqrt{ }^{a}$ & & & & & & & $\sqrt{ }$ \\
\hline Aspergillus novofumigatus CBS 117519 & $\sqrt{ }^{a}$ & & & $\sqrt{ }$ & & & & \\
\hline Aspergillus unilateralis CBS 126.56 & $\sqrt{ }^{a}$ & & & & & & & $\sqrt{ }$ \\
\hline Aspergillus viridinutans CBS 121595 & $V^{a}$ & & & & & & & $\sqrt{ }$ \\
\hline Neosartoryafischeri CBS 316.89 & $\sqrt{ }^{a}$ & & & $\sqrt{ }$ & & $\sqrt{ }$ & & $\sqrt{ }$ \\
\hline Neosartoryahiratsukae CBS 124073 & $\sqrt{ }^{a}$ & & & & & & & $\sqrt{ }$ \\
\hline Neosartoryapseudofischeri CBS $208.92^{\mathrm{b}}$ & $\sqrt{ }^{a}$ & & & & & & & $\sqrt{ }$ \\
\hline Neosartoryaudagawae CBS 114217 & $\sqrt{ }^{a}$ & & $\sqrt{ }$ & & & & & $\sqrt{ }$ \\
\hline
\end{tabular}

a) Unspecific amplification with MC3 primers (confirmed after sequence analysis).

b) Similar results were observed with other tested reference strains.

multiplex panel at $55^{\circ} \mathrm{C}$, as seen in Table 1 . The relevant pathogens of section Fumigati, A. fumigatiaffinis, $N$. fischeri and $N$. udagawae, were clearly distinguished from A. fumigatus and from all the other species within this section. In addition, $A$. novofumigatus was also identified. Besides A. fumigatus isolate, MC6a was uniquely amplified with $N$. fischeri isolate, while MC8 was obtained exclusively with $N$. udagawae. The marker MC5 was amplified with $A$. fumigatiaffinis and $A$. novofumigatus (Table 1). Few microsatellites showed more than three repeat motifs, as it was the case of MC6a in A. lentulus and MC6b in A. unilateralis (sequence analysis of the amplified markers was added as supplementary Table A1). Sequence analysis of marker MC6b showed that $A$. lentulus and $A$. viridinutans (the most relevant species in clinics besides $A$. fumigatus) were different from all the other tested species.

\section{Discussion}

Species such as A. lentulus, A. viridinutans, N. pseudofischeri, and N. udagawae have been described as human pathogens associated to severe cases of trabecular bone invasion, cutaneous, cerebral, liver or pulmonary aspergillosis [1,2,21-23]. In addition, some species were reported as primary resistant in vitro to the substance class of azole antifungals [6,24]. Therefore, due to their intrinsic resistance, infections caused by strains of these species cause difficult to treat infections that deserve increased attention by clinicians. Molecular techniques are recommended for the correct identification of species within the group "A. fumigatus complex", but most clinical laboratories still cannot afford to routinely implement sequencing technologies. Few electrophoretic methodologies are available for molecular identification of A. fumigatus and related species and represent valid alternatives [7-10]. Since genotyping strategies have been strongly recommended by researchers, clinicians and technicians to be implemented in clinical laboratories, it would be desirable to combine both identification and genotyping capabilities in a single method. In this study, we explored the specificity of an A. fumigatus microsatellite genotyping panel in a group of closely related fungal species. The specificity of microsatellite multiplex was confirmed similar to previously described for other standard molecular methodology, such as MLST [4]. In fact, A. fumigatus could be correctly identified employing this strategy, similarly to what was previously described for Candida parapsilosis [18], Cryptococcus neoformans [15], Paracoccidioides brasiliensis [17], and Saccharomyces boulardii [16] when using microsatellite markers combined in a multiplex. It is worth mentioning that simplified methodologies based on restricted genotyping panels of only one or two microsatellite markers [e.g. 25], although more practical and rapid for epidemiological studies, can produce inaccurate results. Our data adds to the increasingly reported application of microsatellite alleles to identify some fungi within complexes of species. In this study we also noticed a low transferability of microsatellites within section Fumigati, namely when comparing $N$. fischeri genome. A small number of markers (4 of 25) have also been described as transferable from related Uredinales species to Hemileia vastatrix [26]. Our results of section Fumigati agree with previous reports that describe a smaller fraction of cross species transfer of microsatellites within fungal genera when compared with higher eukaryotes [27].

Genomic regions of eukaryotes and prokaryotes with microsatellites are prone to genomic alterations particularly insertions and deletions [28]. In this work we observed such modifications when we compared the genomes of $A$. fumigatus and $N$. fischeri in regions with microsatellites. The motif length (tri-, tetra- or pentanucleotide) was not correlated with an increased presence 
in closely related species. Such genomic dynamics resulted in a distinct electrophoretic profile for each species following the application of microsatellite-based PCR multiplex at $55^{\circ} \mathrm{C}$. The relevant pathogens of section Fumigati, such as A. fumigatiaffinis, $N$. fischeri and $N$. udagawae, were easily identified, however, testing this strategy in a broader range of species and isolates would better support identification of species within Aspergillus section Fumigati. This strategy has been successfully tested before in the identification of microsatellite transferability in close related species [29]. Furthermore, the genotyping strategies of less studied species of section Fumigati can now be better approached, as new microsatellite markers have now been proposed for A. unilateralis and $N$. fischeri.

Wide application of typing methodologies can give pertinent information regarding microbial epidemiology, chronic colonization for several patients and effectiveness of antibiotic treatments [11-14]. The initial question on the real specificity of the microsatellite markers selected for $A$. fumigatus genotyping was answered in the present work and it represents a genuine and required improvement for applicability of the methodology. We proved that the proposed panel with eight microsatellites [11] is highly appropriate for genotyping A. fumigatus. Besides genotyping, microsatellite-based multiplex PCR allows the identification of $A$. fumigatus and a slight modification of PCR conditions also allow identifying other pathogenic species within section Fumigati, particularly A. fumigatiaffinis, N. fischeri, and $N$. udagawae. Sequence analysis of marker MC6b showed that $A$. lentulus and $A$. viridinutans were different from all the other tested species.

\section{Methods}

\section{Fungal strains and culture conditions}

A set of fungal isolates described as belonging to Aspergillus section Fumigati was obtained from Centraalbureau voor Schimmelcultures (CBS): the pathogenic moulds Aspergillus fumigatiaffinis (CBS 117186), Aspergillus lentulus (CBS 116880, 117180, 117182, and 117885), Aspergillus viridinutans (CBS 121595), Neosartorya fischeri (CBS 316.89), Neosartorya hiratsukae (CBS 124073), Neosartorya pseudofischeri (CBS 208.92 and 110899), and Neosartorya udagawae (CBS 114217), and two nonpathogenic moulds Aspergillus novofumigatus (CBS 117519) and Aspergillus unilateralis (CBS 126.56). The reference strain $A$. fumigatus ATCC 46645 was also included in the present work, as well as ten different strains of $A$. fumigatus from our collection. Monospore isolates from all the fungal strains were cultured on Sabouraud dextrose agar for 5 days at $30^{\circ} \mathrm{C}$. A sodium hydroxide based method was used to extract DNA from fungal conidia (protocol at http://www.aspergillus.org.uk/ indexhome.htm?secure/laboratory_protocols). Fungal DNA was suspended in $50 \mu \mathrm{l}$ of sterile water and frozen at $-20^{\circ} \mathrm{C}$. Control of the DNA quality was carried out by amplifying and sequencing the $\beta$-tubulin region in all tested fungi, using previously selected primers [10].

\section{Microsatellite-based PCR multiplex}

Microsatellite PCR multiplex was performed according to previously selected primers that allowed the identification of the microsatellites based on tri-, tetra-, and pentanucleotide motifs located in different chromosomes [11]. The PCRs were performed in $5 \mu \mathrm{L}$ final volume, with $1 \mu \mathrm{L}$ of genomic DNA $(1-5 \mathrm{ng} / \mu \mathrm{L}), 2.5 \mu \mathrm{L}$ of $2 \times$ Qiagen multiplex PCR master mixes (Qiagen, Hilden, Germany) and $0.5 \mu \mathrm{L}$ of a mix of eight primer pairs, at $2 \mu \mathrm{M}$ concentration. After a $95^{\circ} \mathrm{C}$ preincubation step of $15 \mathrm{~min}$, PCRs were performed for a total of 30 cycles, using the following conditions: denaturation at $94^{\circ} \mathrm{C}$ for $30 \mathrm{~s}$, annealing at $60^{\circ} \mathrm{C}$ for $90 \mathrm{~s}$ and extension at $72^{\circ} \mathrm{C}$ for $1 \mathrm{~min}$; with a final extension step of $10 \mathrm{~min}$ at $72^{\circ} \mathrm{C}$. The internal size standard GeneScan 500 LIZ (Applied Biosystems, Foster City, CA, USA) $(0.5 \mu \mathrm{L})$ and HiDiformamide (Applied Biosystems) $(12 \mu \mathrm{L})$ were added to the PCR-amplified products and run in an ABI PRISM 3100 genetic analyser 16-capillary electrophoresis system (Applied Biosystems). Fragment size was performed automatically using Genemapper software 4.0 (Applied Biosystems).

\section{DNA sequencing conditions}

PCR-generated fragments were purified with ExoSAP-IT (USB Corporation, Cleveland, Ohio, USA) and the reactions were conducted employing an ABI Big Dye terminator cycle sequencing kit (Applied Biosystems) under the following conditions: after a $95^{\circ} \mathrm{C}$ pre-incubation step of 15 min and DNA denaturation at $96^{\circ} \mathrm{C}$ for $15 \mathrm{~s} ; 35 \mathrm{PCR}$ cycles were performed with primer annealing at $50^{\circ} \mathrm{C}$ for $9 \mathrm{~s}$, an extension at $60^{\circ} \mathrm{C}$ for $2 \mathrm{~min}$; followed by a final extension at $60^{\circ} \mathrm{C}$ for $10 \mathrm{~min}$. A volume of $8 \mu \mathrm{L}$ of $\mathrm{HiDi}-$ formamide were added to the sequencing products and run in an ABI PRISM 3100 Genetic Analyser 16-capillary electrophoresis system. The results were analyzed using the Sequencing 5.2 analysis software (Applied Biosystems).

\section{Data analysis}

Complete genome sequences of $A$. fumigatus AF293 and N. fischeri NRRL 181 available at Ensembl (www. ensembl.org/index.html) were downloaded and the group of eight STRs located in those genomes employing the Geneious software v4.7 (Biomatters Ltd, Auckland, New Zealand) and BioEdit sequence alignment editor (available at http://www.ctu.edu.vn/ dvxe/Bioinformatic/Software/BioEdit.htm). 


\section{Additional files}

\section{Additional file 1: Supplementary Table A1. \\ Additional file 2: Figure A1.}

\section{Competing interest}

No conflicts of interest. The authors have no financial relationship with the organizations that sponsored the research.

\section{Authors' contributions}

RA carried out the experimental studies. RA, AA, and LG conceived the study, participated in its design and coordination and drafted the manuscript. All authors read and approved the final manuscript.

\section{Acknowledgements and funding}

This work was supported by grants from Fundação Calouste Gulbenkian (n. 35-9924-S/2009) and Pfizer Inc. ( ${ }^{\circ}$. IIR\#WS1948668). RA is supported by Fundação para a Ciência e a Tecnologia (FCT) Ciência 2007 and by the European Social Fund. IPATIMUP is an Associate Laboratory of the Portuguese Ministry of Science, Technology and Higher Education and is partially supported by FCT.

Received: 3 May 2012 Accepted: 17 July 2012

Published: 28 July 2012

\section{References}

1. Balajee SA, Gribskov J, Brandt M, Ito J, Fothergill A, Marr KA: Mistaken identity: Neosartoryapseudofischeri and its anamorph masquerading as Aspergillus fumigatus. J ClinMicrobiol 2005, 43:5996-5999.

2. Balajee SA, Gribskov JL, Hanley E, Nickle D, Marr KA: Aspergillus lentulus sp. nov., a new sibling species ofA.fumigatus. Eukaryot Cell 2005, 4:625-632.

3. Samson RA, Hong S, Peterson SW, Frisvad JC, Varga J: Polyphasic taxonomy of AspergillussectionFumigatiand its teleomorph Neosartorya. Stud Mycol 2007, 59:147-203.

4. Balajee SA, Nickle D, Varga J, Marr KA: Molecular studies reveal frequent misidentification of Aspergillus fumigatus by morphotyping. Eukaryot Cell 2006, 5:1705-1712.

5. Hong SB, Shin HD, Hong J, Frisvad JC, Nielsen PV, Varga J, Samson RA: New taxa ofNeosartoryaandAspergillusinAspergillussectionFumigati. Antonie Van Leeuwenhoek 2008, 93:87-98.

6. Yaguchi T, Horie Y, Tanaka R, Matsuzawa T, Ito J, Nishimura K: Molecular phylogenetics of multiple genes onAspergillussectionFumigatiisolated from clinical specimens in Japan. Jap J Med Mycol 2007, 48:37-46.

7. Brandt ME, Padhye AA, Mayer LW, Holloway BP: Utility of random amplified polymorphic DNA PCR and TaqMan automated detection in molecular identification of Aspergillus fumigatus. J ClinMicrobiol 1998, 36:2057-2062

8. Staab JF, Balajee SA, Marr KA: AspergillusSectionFumigati typing by PCR restriction fragment polymorphism. J ClinMicrobiol 2009, 47:2079-2083.

9. Etienne KA, Gade L, Lockhart SR, Diekema DJ, Messer SA, Pfaller MA, Balajee SA: Screening of a large global Aspergillus fumigatus species complex collection by using a species-specific microsphere-based Luminex assay. J Clin Microbiol 2009, 47:4171-4172.

10. Serrano R, Gusmão L, Amorim A, Araujo R: Rapid identification of Aspergillus fumigatus within section Fumigati. BMC Microbiol 2011, 11:82.

11. Araujo R, Pina-Vaz C, Rodrigues AG, Amorim A, Gusmão L: Simple and highly discriminatory microsatellite-based multiplex PCR for Aspergillus fumigatus strain typing. Clin Microbiol Infect 2009, 15:260-266.

12. Amorim A, Guedes-Vaz L, Araujo R: Susceptibility to five antifungals of Aspergillus fumigatus strains isolated from chronically colonised cystic fibrosis patients receiving azole therapy. Int J Antimicrob Agents 2010, 35:396-399.

13. Balajee SA, de Valk HA, Lasker BA, Meis JF, Klaassen CH: Utility of a microsatellite assay for identifying clonally related outbreak isolates of Aspergillus fumigatus. J Microbiol Methods 2008, 73:252-256.

14. Vanhee LM, Symoens F, Bouchara JP, Nelis HJ, Coenye T: High-resolution genotyping of Aspergillus fumigatus isolates recovered from chronically colonised patients with cystic fibrosis. Eur J Clin Microbiol Infect Dis 2008, 27:1005-1007.
15. Hanafy A, Kaocharoen S, Jover-Botella A, Katsu M, lida S, Kogure T, Gonoi T: Mikami Y. Meyer W: Multilocus microsatellite typing for Cryptococcus neoformans var. grubii. Med Mycol 2008, 46:685-696.

16. Hennequin C, Thierry A, Richard GF, Lecointre G, Nguyen HV, Gaillardin C, Dujon B: Microsatellite typing as a new tool for identification of Saccharomyces cerevisiae strains. J Clin Microbiol 2001, 39:551-559.

17. Matute DR, Sepulveda VE, Quesada LM, Goldman GH, Taylor JW, Restrepo A, McEwen JG: Microsatellite analysis of three phylogenetic species of Paracoccidioidesbrasiliensis. J ClinMicrobiol 2006, 44:2153-2157.

18. Sabino R, Sampaio P, Rosado L, Stevens DA, Clemons KV, Pais C: New polymorphic microsatellite markers able to distinguish among Candida parapsilosis sensu stricto isolates. J Clin Microbiol 2010, 48:1677-1682.

19. Kuhls K, Keilonat L, Ochsenreither S, Schaar M, Schweynoch C, Presber W Schönian G: Multilocus microsatellite typing (MLMT) reveals genetically isolated populations between and within the main endemic regions of visceral leishmaniasis. Microb Infect 2007, 9:334-343.

20. Fedorova ND, Khaldi N, Joardar VS, Maiti R, Amedeo P, Anderson MJ, Crabtree J, Silva JC, Badger JH, Albarraq A, Angiuoli S, Bussey H, Bowyer P, Cotty PJ, Dyer PS, Egan A, Galens K, Fraser-Liggett CM, Haas BJ, Inman JM, Kent R, Lemieux S, Malavazi I, Orvis J, Roemer T, Ronning CM, Sundaram JP, Sutton G, Turner G, Venter JC, White OR, Whitty BR, Youngman P, Wolfe KH, Goldman GH, Wortman JR, Jiang B, Denning DW, Nierman WC: Genomic islands in the pathogenic filamentous fungus Aspergillus fumigatus. PLOS Genet 2008, 4:e1000046.

21. Sugui JA, Vinh DC, Nardone G, Shea YR, Chang YC, Zelazny AM, Marr KA, Holland SM, Kwon-Chung KJ: Neosartorya udagawae (Aspergillus udagawae), an emerging agent of aspergillosis: how different is it from Aspergillus fumigatus?. J Clin Microbiol 2010, 48:220-228.

22. Padhye AA, Godfrey JH, Chandler FW, Peterson SW: Osteomyelitis caused by Neosartoryapseudofischeri. J ClinMicrobiol 1994, 32:2832-2836.

23. Guarro J, Kallas EG, Godoy P, Karenina A, Gené J, Stchigel A, Colombo AL: Cerebral aspergillosis caused by Neosartorya hiratsukae, Brazil. Emerg Infect Dis 2002, 8:989-991.

24. Alcazar-Fuoli L, Mellado E, Alastruey-Izquierdo A, Cuenca-Estrella M, Rodriguez-Tudela JL: Aspergillus section Fumigati: antifungal susceptibility patterns and sequence-based identification. Antimicrob Agents Chemother 2008, 52:1244-1251.

25. Barada G, Basma R, Khalaf RA: Microsatellite DNA identification and genotyping of Candida albicans from Lebanese clinical isolates. Mycopathologia 2008, 165:115-125.

26. Cristancho M, Escobar C: Transferability of SSR markers from related Uredinales species to the coffee rust Hemileiavastatrix. Genet Mol Res 2008, 7:1186-1192

27. Baird RE, Wadl PA, Allen T, McNeill D, Wang X, Moulton JK, Rinehart TA, Abbas HK, Shier T, Trigiano RN: Variability of United States isolates of Macrophominaphaseolina based on simple sequence repeats and cross genus transferability to related genera within botryosphaeriaceae. Mycopathologia 2010, 170:169-180.

28. McDonald MJ, Wang WC, Huang HD, Leu JY: Clusters of nucleotide substitutions and insertion/deletion mutations are associated with repeat sequences. PLOS Biol 2011, 9:e1000622.

29. Dutech C, Enjalbert J, Fournier E, Delmotte F, Barrès B, Carlier J, Tharreau D, Giraud T: Challenges of microsatellite isolation in fungi. Fungal Genet Bio 2007, 44:933-949.

\section{doi:10.1186/1471-2180-12-154}

Cite this article as: Araujo et al:: Diversity and specificity of

microsatellites within Aspergillus section Fumigati. BMC Microbiology 2012 $12: 154$ 